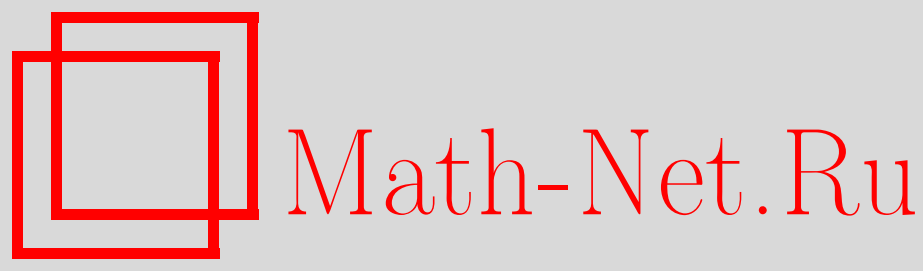

Ю. П. Чубурин, Существование майорановских локализованных состояний в сверхпроводящей нанопроволоке вблизи примеси, ТМФ, 2018, том 197, номер 2, 279-289

DOI: https://doi.org/10.4213/tmf9457

Использование Общероссийского математического портала Math-Net.Ru подразумевает, что вы прочитали и согласны с пользовательским соглашением http://www.mathnet.ru/rus/agreement

Параметры загрузки:

IP: 54.174 .149 .18

26 апреля 2023 г., 08:52:52

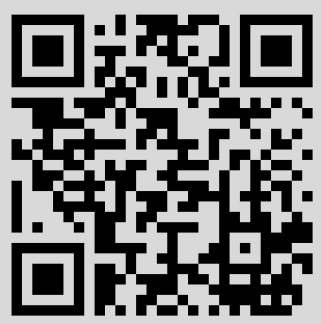




\title{
ФИЗИКА
}

Том 197, № 2

ноябрь, 2018

(C) 2018 г.

Ю. П. Чубурин*

\section{СУЩЕСТВОВАНИЕ МАЙОРАНОВСКИХ ЛОКАЛИЗОВАННЫХ СОСТОЯНИЙ В СВЕРХПРОВОДЯЩЕЙ НАНОПРОВОЛОКЕ ВБЛИЗИ ПРИМЕСИ}

\begin{abstract}
Рассматривается нанопроволока с наведенным в результате эффекта близости $s$-волновым сверхпроводящим порядком в присутствии зеемановского поля и взаимодействия Рашбы. Для малой сверхпроводящей щели и малых импульсов аналитически доказано существование майорановских локализованных состояний при определенном локальном изменении зеемановского поля или сверхпроводящего порядка, а также получены явные выражения для соответствующих волновых функций. Исследовано рассеяние возбужденных состояний с энергиями, близкими к граничным точкам щели, при прохождении через примесь при локальных изменениях указанных параметров системы вблизи этой примеси; показано, что вероятность прохождения равна единице.
\end{abstract}

Ключевые слова: сверхпроводимость, майорановские локализованные состояния, нанопроволока, примесь, зеемановское поле, задача рассеяния.

DOI: https://doi.org/10.4213/tmf9457

\section{1. ВВЕДЕНИЕ}

В последние годы ведется интенсивный экспериментальный поиск майорановских локализованных состояний (МЛС), что вызвано их вероятным использованием в квантовых вычислениях [1]-[3]. МЛС являются квазичастицами с нулевой энергией, возникающими в структурах с индуцированной благодаря эффекту близости сверхпроводимостью. МЛС обладают двумя важными для приложений свойствами [2]-[4]: во-первых, они совпадают со своими античастицами, что делает их устойчивыми к внешним воздействиям; во-вторых, они подчиняются неабелевой квантовой статистике, что открывает перспективы их использования в квантовых компьютерах.

Работа частично поддержана УрО РАН (грант № 15-8-2-12).

* Удмуртский федеральный исследовательский центр Уральского отделения Российской академии наук, Ижевск, Россия. E-mail: chuburin@udman.ru 
Известно [5], что немагнитные примеси в сверхпроводящей $s$-волновой нанопроволоке могут порождать локализованные состояния внутри щели, в том числе МЛС, лишь в присутствии зеемановского поля и спин-орбитального взаимодействия Рашбы. При этом МЛС возникают на границе различных топологических фаз сверхпроводящей структуры, в частности на концах нанопроволоки [2], [6], [7].

В настоящей статье изучается возможность возникновения МЛС в новой ситуации - в окрестности одиночной примеси в нанопроволоке при изменении зеемановского поля или сверхпроводящего порядка вблизи примеси. Для малой сверхпроводящей щели и малых импульсов аналитически доказано существование двух линейно независимых МЛС в этой окрестности в случае локального возмущения указанных параметров системы, но при отсутствии собственно примеси. В статье найдены условия существования МЛС, а также явный вид их волновых функций. Хотя по формальным признакам это действительно МЛС, стандартные МЛС пространственно разделены, и это свойство требуется при их практическом использовании $^{1)}$ [8]. Однако можно ожидать пространственного разделения этих вырожденных состояний на два стандартных МЛС при небольших возмущениях (см. раздел 3 , а также статью [9]).

Кроме того, в статье с целью изучения возможных сопровождающих появление МЛС экспериментально наблюдаемых явлений исследована задача рассеяния возбужденных состояний с энергиями, близкими к границе щели, при описанных выше возмущениях системы. Показано, что вероятность прохождения равна единице, а при наличии примеси возможно локальное увеличение плотности состояний.

\section{2. ФУНКЦИЯ ГРИНА}

В статье рассматривается гамильтониан Боголюбова-де Жена вида [3], [4], [10]

$$
H=\left(\begin{array}{cc}
-\partial_{x}^{2}-i \alpha \partial_{x} \sigma_{y}+M \sigma_{z} & i \Delta \sigma_{y} \\
-i \Delta^{*} \sigma_{y} & -\left(-\partial_{x}^{2}+i \alpha \partial_{x} \sigma_{y}+M \sigma_{z}\right)
\end{array}\right)
$$

описывающий в приближении среднего поля возбуждения (квазичастицы) в одномерной нанопроволоке с наведенным $s$-волновым сверхпроводящим порядком в присутствии зеемановского члена и взаимодействия Рашбы (мы полагаем $\hbar^{2} / 2 m^{*}=1$, где $m^{*}$ - эффективная масса электрона). Здесь $\sigma_{x}, \sigma_{y}$ и $\sigma_{z}$ - матрицы Паули, $\Delta$ - вещественный параметр сверхпроводящего порядка, индуцированного благодаря эффекту близости, $\alpha \neq 0$ - параметр взаимодействия Рашбы и $M \neq 0$ обозначает величину зеемановского поля, порождаемого или внешним магнитным полем, или слоем ферромагнетика. МЛС описываются собственными векторами (возмущенного) гамильтониана Боголюбова-де Жена, отвечающими нулевой энергии и имеющими вид

$$
\psi(x)=\left(\psi_{1}^{\uparrow}(x), \psi_{1}^{\downarrow}(x), \psi_{2}^{\uparrow}(x), \psi_{2}^{\downarrow}(x)\right)^{\mathrm{T}}=\left(\psi_{1}(x), \psi_{1}^{\prime}(x), \psi_{2}(x), \psi_{2}^{\prime}(x)\right)^{\mathrm{T}},
$$

1) В терминологии работы [9] сильно перекрывающиеся МЛС - это "регулярные" андреевские локализованные состояния. В настоящее время актуальной является задача отделить в эксперименте “классические" МЛС от других локализованных состояний с нулевой энергией [8], [9]. 
где стрелка обозначает направление спина, функции с индексом "1" отвечают частицам, а с индексом "2" - дыркам. При этом должно выполняться условие [1]

$$
\psi_{2}^{* *}=-\psi_{1}, \quad \psi_{2}^{*}=\psi_{1}^{\prime},
$$

которое означает, что частица совпадает со своей античастицей.

Для того чтобы иметь возможность получить аналитические результаты, будем рассматривать частицы с малыми импульсами, пренебрегая слагаемыми порядка $p^{\alpha}$, $\alpha>2$.

В дальнейшем нам понадобится резольвента (функция Грина) $(H-E)^{-1}$ гамильтониана $H$ как для поиска МЛС, так и для исследования задачи рассеяния. Применяя к гамильтониану (1) преобразование Фурье

$$
\widehat{\psi}(p)=\frac{1}{\sqrt{2 \pi}} \int_{-\infty}^{\infty} e^{-i p x} \psi(x) d x
$$

и вычитая энергию $E$, получаем в импульсном представлении равенство

$$
\widehat{H}-E=\left(\begin{array}{cccc}
p^{2}+M-E & -i \alpha p & 0 & \Delta \\
i \alpha p & p^{2}-M-E & -\Delta & 0 \\
0 & -\Delta & -p^{2}-M-E & -i \alpha p \\
\Delta & 0 & i \alpha p & -p^{2}+M-E
\end{array}\right)
$$

Определитель матрицы (3) при указанном выше приближении равен

$$
\begin{aligned}
d & =\operatorname{det}(\widehat{H}-E)=E^{4}-2\left(M^{2}+\Delta^{2}+\alpha^{2} p^{2}\right) E^{2}+\left(M^{2}-\Delta^{2}\right)^{2}+2 \alpha^{2} p^{2}\left(M^{2}-\Delta^{2}\right)= \\
& =2 \alpha^{2}\left(M^{2}-\Delta^{2}-E^{2}\right)\left(p^{2}-a^{2}\right),
\end{aligned}
$$

где

$$
a^{2}=-\frac{\left(M^{2}-\Delta^{2}-E^{2}\right)^{2}-4 \Delta^{2} E^{2}}{2 \alpha^{2}\left(M^{2}-\Delta^{2}-E^{2}\right)} .
$$

Равенство $d=0$ согласно формуле (4) записывается в виде

$$
E^{2}=\left(\Delta \pm \sqrt{M^{2}+\alpha^{2} p^{2}}\right)^{2} .
$$

Следовательно, спектр $H$ описывается неравенствами ||$M|-| \Delta|| \leqslant|E|<\infty$. Щель в спектре исчезает, если $|M|=|\Delta|$.

Найдем функцию Грина в импульсном представлении. Для этого решим уравнение $(\widehat{H}-E) \widehat{\psi}=\hat{\varphi}$ относительно $\widehat{\psi}$. Обозначим через $d_{j}$ определитель матрицы $\widehat{H}-E$ после замены $j$-го столбца на столбец $\left(\hat{\varphi}_{1}, \hat{\varphi}_{1}^{\prime}, \hat{\varphi}_{2}, \hat{\varphi}_{2}^{\prime}\right)^{\mathrm{T}}$. С помощью выражений (3)-(5) и формул Крамера находим

$$
\begin{array}{ll}
\widehat{\psi}_{1}=\left((\widehat{H}-E)^{-1} \hat{\varphi}\right)_{1}=\frac{d_{1}}{d}, & \widehat{\psi}_{1}^{\prime}=\left((\widehat{H}-E)^{-1} \hat{\varphi}\right)_{1}^{\prime}=\frac{d_{2}}{d}, \\
\widehat{\psi}_{2}=\left((\widehat{H}-E)^{-1} \hat{\varphi}\right)_{2}=\frac{d_{3}}{d}, & \widehat{\psi}_{2}^{\prime}=\left((\widehat{H}-E)^{-1} \hat{\varphi}\right)_{2}^{\prime}=\frac{d_{4}}{d},
\end{array}
$$


где $d$ взято из (4),

$$
\begin{aligned}
d_{1}= & \left(\left(-M^{2}+\Delta^{2}-E^{2}-2 E M+\alpha^{2}(M-E)\right) p^{2}+\left(M^{2}-E^{2}\right)(M+E)-\right. \\
& \left.-\Delta^{2}(M-E)\right) \hat{\varphi}_{1}-i \alpha p\left(M^{2}-\Delta^{2}-E^{2}\right) \hat{\varphi}_{1}^{\prime}-2 i \alpha p E \Delta \hat{\varphi}_{2}- \\
& -\Delta\left(M^{2}-\Delta^{2}+E^{2}+2 M E+\alpha^{2} p^{2}\right) \hat{\varphi}_{2}^{\prime}, \\
d_{2}= & i \alpha p\left(M^{2}-\Delta^{2}-E^{2}\right) \hat{\varphi}_{1}+ \\
& +\left(\left(-M^{2}+\Delta^{2}-E^{2}+2 E M+\alpha^{2}(M-E)\right) p^{2}-\left(M^{2}-E^{2}\right)(M-E)+\right. \\
& \left.+\Delta^{2}(M+E)\right) \hat{\varphi}_{1}^{\prime}+\Delta\left(M^{2}-\Delta^{2}+E^{2}-2 M E+\alpha^{2} p^{2}\right) \hat{\varphi}_{2}-2 i \alpha p E \Delta \hat{\varphi}_{2}^{\prime}, \\
d_{3}= & 2 i \alpha p E \Delta \hat{\varphi}_{1}+\Delta\left(M^{2}-\Delta^{2}+E^{2}-2 M E+\alpha^{2} p^{2}\right) \hat{\varphi}_{1}^{\prime}+ \\
& +\left(\left(M^{2}-\Delta^{2}+E^{2}-2 E M-\alpha^{2}(M-E)\right) p^{2}-\right. \\
& \left.-\left(M^{2}-E^{2}\right)(M-E)+\Delta^{2}(M+E)\right) \widehat{\varphi}_{2}-i \alpha p\left(M^{2}-\Delta^{2}-E^{2}\right) \hat{\varphi}_{2}^{\prime}, \\
d_{4}= & -\Delta\left(M^{2}-\Delta^{2}+E^{2}+2 M E+\alpha^{2} p^{2}\right) \hat{\varphi}_{1}+ \\
& +2 i \alpha p E \Delta \hat{\varphi}_{1}^{\prime}+i \alpha p\left(M^{2}-\Delta^{2}+E^{2}\right) \hat{\varphi}_{2}+ \\
& +\left(\left(M^{2}-\Delta^{2}+E^{2}+2 E M+\alpha^{2}(M+E)\right) p^{2}+\right. \\
& \left.+\left(M^{2}-E^{2}\right)(M+E)-\Delta^{2}(M-E)\right) \hat{\varphi}_{2}^{\prime} .
\end{aligned}
$$

\section{3. СУЩЕСТВОВАНИЕ МАЙОРАНОВСКИХ СОСТОЯНИЙ}

Для проверки существования МЛС вблизи примеси используем уравнение

$$
\psi=-(H-E)^{-1} V \psi
$$

для $E=0$, где $V$ - сепарабельный потенциал, действующий вблизи нуля и определяемый равенством

$$
V \psi(x)=\left(\begin{array}{c}
\left(V_{0}+m\right)\left(\psi_{1}, \varphi_{0}\right)+\delta\left(\psi_{2}^{\prime}, \varphi_{0}\right) \\
\left(V_{0}-m\right)\left(\psi_{1}^{\prime}, \varphi_{0}\right)-\delta\left(\psi_{2}, \varphi_{0}\right) \\
-\delta\left(\psi_{1}^{\prime}, \varphi_{0}\right)-\left(V_{0}+m\right)\left(\psi_{2}, \varphi_{0}\right) \\
\delta\left(\psi_{1}, \varphi_{0}\right)+\left(-V_{0}+m\right)\left(\psi_{2}^{\prime}, \varphi_{0}\right)
\end{array}\right) \varphi_{0}(x)
$$

(по поводу сепарабельных потенциалов см., например, работы [11]-[13]). Здесь $V_{0}$ описывает примесь, $m$ и $\delta$ являются локальными возмущениями зеемановского поля и параметра сверхпроводимости соответственно (см. вид гамильтониана (3)), $(\psi, \varphi)=\int_{-\infty}^{\infty} \psi(x) \overline{\varphi(x)} d x$ - скалярное произведение и $\varphi_{0}-$ четная неотрицательная функция такая, что $\varphi_{0} \neq 0$ только в достаточно малой $\varepsilon$-окрестности нуля и $\int_{-\infty}^{\infty} \varphi_{0}(x) d x=1$. Для функций $\psi$, которые мало меняются в окрестности нуля (в том числе для рассматриваемых ниже волновых функций), имеем

$$
\left(\psi, \varphi_{0}\right) \varphi_{0} \approx \psi(0) \delta(x)
$$

где $\delta(x)-\delta$-функция Дирака, т. е. рассматриваемый сепарабельный потенциал действует подобно $\delta$-образному потенциалу (заметим, что использование потенциала с нулевым радиусом действия здесь невозможно, поскольку в силу специфики функции Грина возникает выражение $\delta^{2}(x)$, не имеющее смысла).

В дальнейшем будем предполагать, что $M, \Delta>0$ и $|M-\Delta| \ll M, \alpha^{2}$. Тогда для $E=0$ получаем из (5) равенство $a^{2}=-\left(M^{2}-\Delta^{2}\right) /\left(2 \alpha^{2}\right)$. Для убывания волновой 
функции МЛС нужно (см. ниже вид функции Грина (11)) наложить условие $M>\Delta$, что отвечает нетривиальной топологической фазе [3], и выбрать знак

$$
a=\frac{i \sqrt{M^{2}-\Delta^{2}}}{\sqrt{2}|\alpha|} .
$$

Используя выражения (6), (7) и пренебрегая согласно сделанным выше предположениям слагаемыми порядка $M-\Delta$ в коэффициентах при $\hat{\varphi}_{1,2}^{(\prime)}$, перепишем функцию Грина для $E=0$ :

$$
\begin{aligned}
\widehat{\psi}_{1}(p) & =\left(\widehat{H}^{-1} \hat{\varphi}\right)_{1}(p)=\frac{p^{2}\left(M \hat{\varphi}_{1}(p)-\Delta \hat{\varphi}_{2}^{\prime}(p)\right)}{2\left(M^{2}-\Delta^{2}\right)\left(p^{2}-a^{2}\right)}, \\
\widehat{\psi}_{1}^{\prime}(p) & =\left(\widehat{H}^{-1} \widehat{\varphi}\right)_{1}^{\prime}(p)=-\frac{p^{2}\left(M \hat{\varphi}_{1}^{\prime}(p)-\Delta \hat{\varphi}_{2}(p)\right)}{2\left(M^{2}-\Delta^{2}\right)\left(p^{2}-a^{2}\right)}, \\
\widehat{\psi}_{2}(p) & =\left(\widehat{H}^{-1} \hat{\varphi}\right)_{2}(p)=-\frac{p^{2}\left(M \hat{\varphi}_{2}(p)-\Delta \hat{\varphi}_{1}^{\prime}(p)\right)}{2\left(M^{2}-\Delta^{2}\right)\left(p^{2}-a^{2}\right)} \\
\widehat{\psi}_{2}^{\prime}(p) & =\left(\widehat{H}^{-1} \hat{\varphi}\right)_{2}^{\prime}(p)=\frac{p^{2}\left(M \hat{\varphi}_{2}^{\prime}(p)-\Delta \hat{\varphi}_{1}(p)\right)}{2\left(M^{2}-\Delta^{2}\right)\left(p^{2}-a^{2}\right)}
\end{aligned}
$$

С помощью известной формулы

$$
\frac{1}{\sqrt{2 \pi}} \int_{-\infty}^{\infty} \frac{e^{i p x} \hat{\varphi}(p) d p}{p^{2}-a^{2}}=-\frac{1}{2 i a} \int_{-\infty}^{\infty} e^{i a\left|x-x^{\prime}\right|} \varphi\left(x^{\prime}\right) d x^{\prime}
$$

получаем из формул (10) функцию Грина в координатном представлении:

$$
\begin{aligned}
\psi_{1}(x)= & \left(H^{-1} \varphi\right)_{1}(x)=\frac{M \varphi_{1}(x)-\Delta \varphi_{2}^{\prime}(x)}{2\left(M^{2}-\Delta^{2}\right)}- \\
& -\frac{a}{4 i\left(M^{2}-\Delta^{2}\right)} \int_{-\infty}^{\infty} e^{i a\left|x-x^{\prime}\right|}\left(M \varphi_{1}\left(x^{\prime}\right)-\Delta \varphi_{2}^{\prime}\left(x^{\prime}\right)\right) d x^{\prime}, \\
\psi_{1}^{\prime}(x)= & \left(H^{-1} \varphi\right)_{1}^{\prime}(x)=-\frac{M \varphi_{1}^{\prime}(x)-\Delta \varphi_{2}(x)}{2\left(M^{2}-\Delta^{2}\right)}+ \\
& +\frac{a}{4 i\left(M^{2}-\Delta^{2}\right)} \int_{-\infty}^{\infty} e^{i a\left|x-x^{\prime}\right|}\left(M \varphi_{1}^{\prime}\left(x^{\prime}\right)-\Delta \varphi_{2}\left(x^{\prime}\right)\right) d x^{\prime}, \\
\psi_{2}(x)= & \left(H^{-1} \varphi\right)_{2}(x)=-\frac{M \varphi_{2}(x)-\Delta \varphi_{1}^{\prime}(x)}{2\left(M^{2}-\Delta^{2}\right)}+ \\
& +\frac{a}{4 i\left(M^{2}-\Delta^{2}\right)} \int_{-\infty}^{\infty} e^{i a\left|x-x^{\prime}\right|}\left(M \varphi_{2}\left(x^{\prime}\right)-\Delta \varphi_{1}^{\prime}\left(x^{\prime}\right)\right) d x^{\prime}, \\
\psi_{2}^{\prime}(x)= & \left(H^{-1} \varphi\right)_{2}^{\prime}(x)=\frac{M_{2}^{\prime}(x)-\Delta \varphi_{1}(x)}{2\left(M^{2}-\Delta^{2}\right)}- \\
& -\frac{a}{4 i\left(M^{2}-\Delta^{2}\right)} \int_{-\infty}^{\infty} e^{i a\left|x-x^{\prime}\right|}\left(M \varphi_{2}^{\prime}\left(x^{\prime}\right)-\Delta \varphi_{1}\left(x^{\prime}\right)\right) d x^{\prime} .
\end{aligned}
$$


Согласно соотношениям (9), (11) запишем уравнение (8) для $E=0$ в виде

$$
\begin{aligned}
\psi_{1}(x)= & \frac{-1}{2\left(M^{2}-\Delta^{2}\right)}\left(\left(M\left(V_{0}+m\right)-\Delta \delta\right) \psi_{1}+\left(\Delta\left(V_{0}-m\right)+M \delta\right) \psi_{2}^{\prime}, \varphi_{0}\right) \varphi_{0}(x)+ \\
& +\frac{a}{4 i\left(M^{2}-\Delta^{2}\right)} \int_{-\infty}^{\infty} e^{i a\left|x-x^{\prime}\right|}\left(\left(M\left(V_{0}+m\right)-\Delta \delta\right) \psi_{1}+\right. \\
& \left.+\left(\Delta\left(V_{0}-m\right)+M \delta\right) \psi_{2}^{\prime}, \varphi_{0}\right) \varphi_{0}\left(x^{\prime}\right) d x^{\prime}, \\
\psi_{1}^{\prime}(x)= & \frac{1}{2\left(M^{2}-\Delta^{2}\right)}\left(\left(M\left(V_{0}-m\right)+\Delta \delta\right) \psi_{1}^{\prime}+\left(\Delta\left(V_{0}+m\right)-M \delta\right) \psi_{2}, \varphi_{0}\right) \varphi_{0}(x)- \\
& -\frac{a}{4 i\left(M^{2}-\Delta^{2}\right)} \int_{-\infty}^{\infty} e^{i a\left|x-x^{\prime}\right|}\left(\left(M\left(V_{0}-m\right)+\Delta \delta\right) \psi_{1}^{\prime}+\right. \\
& \left.+\left(\Delta\left(V_{0}+m\right)-M \delta\right) \psi_{2}, \varphi_{0}\right) \varphi_{0}\left(x^{\prime}\right) d x^{\prime}, \\
\psi_{2}(x)= & \frac{-1}{2\left(M^{2}-\Delta^{2}\right)}\left(\left(\Delta\left(V_{0}-m\right)+M \delta\right) \psi_{1}^{\prime}+\left(M\left(V_{0}+m\right)-\Delta \delta\right) \psi_{2}, \varphi_{0}\right) \varphi_{0}(x)+ \\
& +\frac{a}{4 i\left(M^{2}-\Delta^{2}\right)} \int_{-\infty}^{\infty} e^{i a\left|x-x^{\prime}\right|}\left(\left(\Delta\left(V_{0}-m\right)+M \delta\right) \psi_{1}^{\prime}+\right. \\
& \left.+\left(M\left(V_{0}+m\right)-\Delta \delta\right) \psi_{2}, \varphi_{0}\right) \varphi_{0}\left(x^{\prime}\right) d x^{\prime}, \\
\psi_{2}^{\prime}(x)= & \frac{1}{2\left(M^{2}-\Delta^{2}\right)}\left(\left(\Delta\left(V_{0}+m\right)-M \delta\right) \psi_{1}+\left(M\left(V_{0}-m\right)+\Delta \delta\right) \psi_{2}^{\prime}, \varphi_{0}\right) \varphi_{0}(x)+ \\
& +\frac{-a}{4 i\left(M^{2}-\Delta^{2}\right)} \int_{-\infty}^{\infty} e^{i a\left|x-x^{\prime}\right|}\left(\left(\Delta\left(V_{0}+m\right)-M \delta\right) \psi_{1}+\right. \\
& \left.+\left(M\left(V_{0}-m\right)+\Delta \delta\right) \psi_{2}^{\prime}, \varphi_{0}\right) \varphi_{0}\left(x^{\prime}\right) d x^{\prime} .
\end{aligned}
$$

\section{Положим}

$$
\begin{aligned}
x_{j}^{\left({ }^{\prime}\right)} & =\left(\psi_{j}^{\left({ }^{\prime}\right)}, \varphi_{0}\right), \quad j=1,2, \quad c=\int_{-\infty}^{\infty} \varphi_{0}^{2}(x) d x, \\
c^{\prime} & =\int_{-\infty}^{\infty} \int_{-\infty}^{\infty} e^{i a\left|x-x^{\prime}\right|} \varphi_{0}(x) \varphi_{0}\left(x^{\prime}\right) d x d x^{\prime} .
\end{aligned}
$$

Заметим, что в силу неравенства Коши-Буняковского

$$
1=\int_{-\infty}^{\infty} \varphi_{0}(x) d x \leqslant \sqrt{\int_{-\infty}^{\infty} \varphi_{0}^{2}(x) d x} \sqrt{\int_{-\varepsilon}^{\varepsilon} d x}=\sqrt{2 c \varepsilon}
$$

откуда $c \geqslant(2 \varepsilon)^{-1}$. Скалярно умножая уравнения (12) на $\varphi_{0}$ и интегрируя по $x$, получаем систему

$$
\begin{aligned}
& x_{1}=\frac{\left(\left(M\left(V_{0}+m\right)-\Delta \delta\right) x_{1}+\left(\Delta\left(V_{0}-m\right)+M \delta\right) x_{2}^{\prime}\right)\left(-2 i c+a c^{\prime}\right)}{4 i\left(M^{2}-\Delta^{2}\right)}, \\
& x_{1}^{\prime}=\frac{\left(\left(M\left(V_{0}-m\right)+\Delta \delta\right) x_{1}^{\prime}+\left(\Delta\left(V_{0}+m\right)-M \delta\right) x_{2}\right)\left(2 i c-a c^{\prime}\right)}{4 i\left(M^{2}-\Delta^{2}\right)}, \\
& x_{2}=\frac{\left(\left(\Delta\left(V_{0}-m\right)+M \delta\right) x_{1}^{\prime}+\left(M\left(V_{0}+m\right)-\Delta \delta\right) x_{2}\right)\left(-2 i c+a c^{\prime}\right)}{4 i\left(M^{2}-\Delta^{2}\right)}, \\
& x_{2}^{\prime}=\frac{\left(\left(\Delta\left(V_{0}+m\right)-M \delta\right) x_{1}+\left(M\left(V_{0}-m\right)+\Delta \delta\right) x_{2}^{\prime}\right)\left(2 i c-a c^{\prime}\right)}{4 i\left(M^{2}-\Delta^{2}\right)} .
\end{aligned}
$$


Положим, считая, что $\varepsilon$ или $a \sim \sqrt{M-\Delta}$ достаточно малы,

$$
\sigma=\frac{4 i\left(M^{2}-\Delta^{2}\right)}{a c^{\prime}-2 i c} \approx-\frac{2\left(M^{2}-\Delta^{2}\right)}{c} .
$$

Система (13) распадается на две независимые подсистемы

$$
\begin{array}{r}
\left(\begin{array}{cc}
-\sigma+M\left(V_{0}+m\right)-\Delta \delta & \Delta\left(V_{0}-m\right)+M \delta \\
-\Delta\left(V_{0}+m\right)+M \delta & -\sigma-M\left(V_{0}-m\right)-\Delta \delta
\end{array}\right)\left(\begin{array}{l}
x_{1} \\
x_{2}^{\prime}
\end{array}\right)=0 \\
\left(\begin{array}{cc}
\sigma+M\left(V_{0}-m\right)+\Delta \delta & \Delta\left(V_{0}+m\right)-M \delta \\
-\Delta\left(V_{0}-m\right)-M \delta & \sigma-M\left(V_{0}+m\right)+\Delta \delta
\end{array}\right)\left(\begin{array}{l}
x_{1}^{\prime} \\
x_{2}
\end{array}\right)=0
\end{array}
$$

Условие существования ненулевого решения каждой из систем (14) имеет вид (отбрасываем в силу малости $\sigma^{2}$ и пользуемся условием $M \approx \Delta$ )

$$
V_{0}^{2}=(m-\delta)\left(m+\delta+\frac{4 \Delta}{c}\right) .
$$

Если равенство (15) выполнено, то существуют ненулевые решения подсистем (14), которые порождают решения системы (13):

$$
\left.\left(x_{1}, x_{1}^{\prime}, x_{2}, x_{2}^{\prime}\right)=\left(-\sigma-M\left(V_{0}-m\right)-\Delta \delta, 0,0, \Delta\left(V_{0}+m\right)-M \delta\right)\right)
$$

и

$$
\left(x_{1}, x_{1}^{\prime}, x_{2}, x_{2}^{\prime}\right)=\left(0, \sigma-M\left(V_{0}+m\right)+\Delta \delta, \Delta\left(V_{0}-m\right)+M \delta, 0\right) .
$$

Следовательно, если выполнено равенство (15), то существуют два линейно независимых локализованных состояния с нулевой энергией, волновые функции которых находим с помощью формул (12), (16), (17), отбрасывая слагаемое с $a c^{\prime} \sim \sqrt{M-\Delta}$ $\left(c^{\prime} \rightarrow 1\right.$ при $\left.\varepsilon \rightarrow 0\right):$

$$
\begin{aligned}
& \psi_{1}(x)=C\left(\left(-V_{0}+m-\delta, 0,0, V_{0}+m-\delta\right) \varphi_{0}(x), \quad C=\right.\text { const. } \\
& \psi_{2}(x)=C\left(0, V_{0}+m-\delta,-V_{0}+m-\delta, 0\right) \varphi_{0}(x),
\end{aligned}
$$

Для того чтобы функции (18) описывали МЛС, в силу условия (2) требуется выполнение одного из равенств $-V_{0}=0$ или $m-\delta=0$. Но $m-\delta \neq 0$, так как в противном случае согласно $(15) V_{0}=0$ и вследствие $(18) \psi_{1,2}(x)=0$. Следовательно, условия существования МЛС имеют вид

$$
V_{0}=0, \quad m+\delta=-\frac{4 \Delta}{c}, \quad m-\delta \neq 0,
$$

при этом

$$
\psi_{1}(x)=C(1,0,0,1) \varphi_{0}(x), \quad \psi_{2}(x)=C(0,1,1,0) \varphi_{0}(x) .
$$

Например, если $\delta=0$, то $m=-4 \Delta / c$ и последнее условие в (19) выполнено автоматически. Таким образом, для появления МЛС достаточно локального воздействия зеемановского поля и отсутствия обычной примеси.

Описанные выше МЛС отличаются от обычных тем, что имеют кратность два, что делает весьма сомнительным их практическое применение [8]. Однако можно ожидать, что при уширении области действия потенциала (фактически, локального поля Зеемана) эти МЛС пространственно разделяются. Действительно, вследствие 
малости щели можно добиться того, чтобы при уширении локального зеемановского поля, кроме того, менялся знак $M-\Delta+m$ вблизи граничных точек области действия поля. Это приводит к изменению топологической фазы между этими точками [3] с нетривиальной на тривиальную. Вблизи таких точек располагаются МЛС [2], [6], [7]. Но по теореме Руше небольшие изменения параметров системы не влияют на число полюсов (с учетом кратности), поэтому имеющийся полюс второго порядка должен разделиться на два полюса первого порядка вблизи граничных точек области действия локального поля (см. также работу [9]).

Заметим, что в статье [14] рассматривается аналогичная задача существования МЛС вблизи примеси для граничных состояний двумерного топологического изолятора при наличии зеемановского поля. Однако в используемом там гамильтониане выбран неверный знак для дырок. Основной результат для исправленного гамильтониана выглядит почти так же: если $W= \pm v / 2$, где $W$ - среднее от потенциала примеси, а $v$ - скорость граничных состояний, то в окрестности примеси существует МЛС кратности 2, но, в отличие от [14], в любой топологической фазе.

Из вида волновых функций (20) следует, что локализация МЛС не зависит от размера щели; это согласуется с результатами статьи [15], но отличается от ситуации на границе топологического изолятора [14].

Рассмотрим достаточно реальную ситуацию, выбирая значения параметров близкими к экспериментальным для сверхпроводника InSb [16]. Вернем в гамильтониан (1) величины $m^{*}, \hbar$ и положим

$$
\Delta^{\prime}=\left(\frac{\hbar^{2}}{2 m^{*}}\right) \Delta=0.25 \text { мэВ, } \quad \alpha^{\prime}=\left(\frac{\hbar^{2}}{2 m^{*}}\right) \alpha=20 \text { мэВ·нм, }
$$

где $m^{*}=0.015 m_{\mathrm{e}}$. Далее полагаем $M^{\prime}=\left(\hbar^{2} /\left(2 m^{*}\right)\right) M=0.26$ мэВ; здесь $M=$ $g \mu_{\mathrm{B}} B / 2$, где $g=40$ - множитель Ланде, $\mu_{\mathrm{B}}$ - магнетон Бора, $B$ - внешнее магнитное поле. Предположим для простоты, что $\varphi_{0}(x)=h$ для $|x| \leqslant \varepsilon$ и $\varphi_{0}(x)=0$ в противном случае. Тогда $2 h \varepsilon=1, c=2 h^{2} \varepsilon=h=1 /(2 \varepsilon)$. Для $|x|,\left|x^{\prime}\right| \leqslant \varepsilon=1$ нм получаем (см. начало раздела 3)

$$
1-e^{i a\left|x-x^{\prime}\right|} \approx-i a\left|x-x^{\prime}\right|=\frac{\sqrt{M^{\prime 2}-\Delta^{\prime 2}}\left|x-x^{\prime}\right|}{\sqrt{2}\left|\alpha^{\prime}\right|} \leqslant \frac{2 \varepsilon \sqrt{M^{\prime 2}-\Delta^{\prime 2}}}{\sqrt{2}\left|\alpha^{\prime}\right|} \approx 5 \cdot 10^{-3},
$$

которой достаточно для сделанных в этом разделе приближений. Условие существования МЛС в случае $\delta=0$ согласно (20) имеет вид $m^{\prime}=-4 \Delta^{\prime} / c=-4 \Delta^{\prime}(2 \varepsilon) \approx$ -2 мэВ. Заметим, что $2 \varepsilon$ здесь есть длина промежутка локального изменения параметра $M^{\prime}$ на величину $m^{\prime}$.

\section{4. РАССЕЯНИЕ ВБЛИЗИ ГРАНИЦЫ СВЕРХПРОВОДЯЩЕЙ ЩЕЛИ}

С целью обнаружения возможной связи рассеяния для энергий вне щели с существованием МЛС исследуем задачу рассеяния для возбуждений с энергиями

$$
E=M-\Delta+\gamma(M-\Delta)^{2}
$$

где $\gamma>0$. В этом случае формула (5) приводит к равенству

$$
a=\frac{(M-\Delta) \sqrt{2 \gamma \Delta^{2}}}{|\alpha|}
$$

(выбранный знак отвечает движению частицы слева направо). 
Найдем решение $\psi_{0}(x)$ уравнения $(H-E) \psi_{0}(x)=0$, отвечающее налетающему электрону, полагая $\psi_{0}(x)=\widehat{\psi} e^{i a x}$, где $\widehat{\psi}=\left(\widehat{\psi}_{1}, \widehat{\psi}_{1}^{\prime}, \widehat{\psi}_{2}, \widehat{\psi}_{2}^{\prime}\right)^{\mathrm{T}}=$ const удовлетворяет уравнению $(\widehat{H}-E) \widehat{\psi}=0$. Величины $a, E$ из формул $(21),(22)$, имеющие порядок малости $M-\Delta$, заменяем нулем и легко находим, что

$$
\psi_{0}(x)=(1,0,0,-1) \frac{e^{i a x}}{\sqrt{2}}
$$

или

$$
\psi_{0}(x)=(0,1,-1,0) \frac{e^{i a x}}{\sqrt{2}} .
$$

Заменяя в (4) $E$ на выражение из (21), получаем, пренебрегая слагаемыми порядка $(M-\Delta)^{3}$, равенство

$$
d=-(M-\Delta)^{4}
$$

Далее, подставляя в (7) $E$ из $(21)$ и пренебрегая слагаемыми порядка $(M-\Delta)^{2}$ в коэффициентах при $\hat{\varphi}_{1,2}^{(\prime)}$, находим

$$
\begin{array}{ll}
d_{1}=4 \Delta^{2}(M-\Delta)\left(\hat{\varphi}_{1}-\hat{\varphi}_{2}^{\prime}\right), & d_{2}=-\Delta(M-\Delta)^{2}\left(\hat{\varphi}_{1}^{\prime}+\hat{\varphi}_{2}\right), \\
d_{3}=-\Delta(M-\Delta)^{2}\left(\hat{\varphi}_{1}^{\prime}+\hat{\varphi}_{2}\right), & d_{4}=-4 \Delta^{2}(M-\Delta)\left(\hat{\varphi}_{1}-\hat{\varphi}_{2}^{\prime}\right) .
\end{array}
$$

Для функции Грина вместо (13) теперь согласно выражениям (25), (26) имеем

$$
\begin{aligned}
& \psi_{1}(x)=\left((H-E)^{-1} \varphi\right)_{1}(x)=-\frac{4 \Delta^{2}\left(\varphi_{1}-\varphi_{2}^{\prime}\right)}{(M-\Delta)^{3}}, \\
& \psi_{1}^{\prime}(x)=\left((H-E)^{-1} \varphi\right)_{1}^{\prime}(x)=\frac{\Delta\left(\varphi_{1}^{\prime}+\varphi_{2}\right)}{(M-\Delta)^{2}}, \\
& \psi_{2}(x)=\left((H-E)^{-1} \varphi\right)_{2}(x)=\frac{\Delta\left(\varphi_{1}^{\prime}+\varphi_{2}\right)}{(M-\Delta)^{2}}, \\
& \psi_{2}^{\prime}(x)=\left((H-E)^{-1} \varphi\right)_{2}^{\prime}(x)=\frac{4 \Delta^{2}\left(\varphi_{1}-\varphi_{2}^{\prime}\right)}{(M-\Delta)^{3}} .
\end{aligned}
$$

Используя формулы (9), (23), (24), (27), запишем уравнение Липпмана-Швингера, которое распадается на две линейные системы

$$
\begin{aligned}
& \psi_{1}(x)=\frac{e^{i a x}}{\sqrt{2}}+\frac{4 \Delta^{2}}{(M-\Delta)^{3}}\left(\left(V_{0}+m-\delta\right) x_{1}+\left(V_{0}-m+\delta\right) x_{2}^{\prime}\right) \varphi_{0}(x), \\
& \psi_{2}^{\prime}(x)=-\frac{e^{i a x}}{\sqrt{2}}-\frac{4 \Delta^{2}}{(M-\Delta)^{3}}\left(\left(V_{0}+m-\delta\right) x_{1}+\left(V_{0}-m+\delta\right) x_{2}^{\prime}\right) \varphi_{0}(x)
\end{aligned}
$$

и

$$
\begin{aligned}
& \psi_{1}^{\prime}(x)=\frac{e^{i a x}}{\sqrt{2}}-\frac{\Delta}{(M-\Delta)^{2}}\left(\left(V_{0}-m-\delta\right) x_{1}^{\prime}-\left(V_{0}+m+\delta\right) x_{2}\right) \varphi_{0}(x), \\
& \psi_{2}^{\prime}(x)=-\frac{e^{i a x}}{\sqrt{2}}-\frac{\Delta}{(M-\Delta)^{2}}\left(\left(V_{0}-m-\delta\right) x_{1}^{\prime}-\left(V_{0}+m+\delta\right) x_{2}\right) \varphi_{0}(x)
\end{aligned}
$$

Слагаемые с $\varphi_{0}(x)$ не оказывают влияния на вероятности прохождения и отражения, в обоих случаях имеет место полное прохождение независимо от наличия МЛС. 
Однако имеет место более тонкий эффект. Из выражений (28), (29), как и выше, получаем системы

$$
\begin{aligned}
& \left(\begin{array}{cc}
(M-\Delta)^{3} /\left(4 \Delta^{2}\right)-\left(V_{0}+m-\delta\right) c & -\left(V_{0}-m+\delta\right) c \\
\left(V_{0}+m-\delta\right) c & (M-\Delta)^{3} /\left(4 \Delta^{2}\right)+\left(V_{0}-m+\delta\right) c
\end{array}\right)\left(\begin{array}{l}
x_{1} \\
x_{2}^{\prime}
\end{array}\right)= \\
& =\frac{(M-\Delta)^{3}}{4 \sqrt{2} \Delta^{2}}\left(\begin{array}{r}
1 \\
-1
\end{array}\right), \\
& \left(\begin{array}{cc}
(M-\Delta)^{2} / \Delta+\left(V_{0}-m-\delta\right) c & -\left(V_{0}+m+\delta\right) c \\
\left(V_{0}-m-\delta\right) c & (M-\Delta)^{2} / \Delta-\left(V_{0}+m+\delta\right) c
\end{array}\right)\left(\begin{array}{l}
x_{1} \\
x_{2}^{\prime}
\end{array}\right)= \\
& =\frac{(M-\Delta)^{2}}{\sqrt{2} \Delta}\left(\begin{array}{r}
1 \\
-1
\end{array}\right) .
\end{aligned}
$$

Находя из системы (30) $x_{1}, x_{2}^{\prime}$ и подставляя их в $(28)$, получаем, что

$$
\psi_{1}(x)=\frac{e^{i a x}}{\sqrt{2}}-\frac{\varphi_{0}(x)}{\sqrt{2} c}, \quad \psi_{2}^{\prime}(x)=-\frac{e^{i a x}}{\sqrt{2}}+\frac{\varphi_{0}(x)}{\sqrt{2} c} .
$$

Аналогично, из выражений (29), (31) имеем

$$
\psi_{1}^{\prime}(x)=\frac{e^{i a x}}{\sqrt{2}}+\frac{4 V_{0} \Delta \varphi_{0}(x)}{\sqrt{2}(M-\Delta)(m-\delta) c}, \quad \psi_{2}(x)=-\frac{e^{i a x}}{\sqrt{2}}+\frac{4 V_{0} \Delta \varphi_{0}(x)}{\sqrt{2}(M-\Delta)(m-\delta) c} .
$$

Волновые пакеты, отвечающие в равенствах (32), (33) экспонентам, при $t \rightarrow \infty$ уходят, расплываясь, на бесконечность, в то время как волновые пакеты, отвечающие слагаемым с $\varphi_{0}(x)$ (не зависящим от $p=a$ ), находятся на одном и том же месте, но коэффициенты перед ними стремятся к нулю. При этом с самого начала эти коэффициенты в (32) очень малы, а в (33) при наличии примеси весьма велики, так что распад соответствующих локализованных состояний происходит (также ввиду малых импульсов) достаточно медленно, что может означать увеличение плотности состояний вблизи верхней границы щели. Если существуют МЛС, то в силу (19) примесь должна отсутствовать, и этот эффект пропадает.

Заметим, что случай рассеяния для энергии $E=-\left(M-\Delta+\gamma(M-\Delta)^{2}\right)$ сводится к тому, что пары $\left(\psi_{1}(x), \psi_{2}^{\prime}(x)\right)$ и $\left(\psi_{1}^{\prime}(x), \psi_{2}(x)\right)$ меняются местами.

\section{5. ЗАКЛЮЧЕНИЕ}

Как известно, МЛС существуют на концах нанопроволоки с порожденной эффектом близости $s$-волновой сверхпроводимостью при наличии зеемановского поля и взаимодействия Рашбы. В статье аналитически доказано существование МЛС в новой ситуации: для малой сверхпроводящей щели и малых импульсов МЛС могут появиться в топологически нетривиальной фазе при локальном возмущении зеемановского поля или сверхпроводящего порядка. Получены условия существования МЛС, а также явный вид их волновых функций. Также исследована задача рассеяния для энергий вблизи граничных точек сверхпроводящей щели при возможном наличии примеси и локальном изменении упомянутых выше параметров; имеет место полное прохождение, а в присутствии примеси возможно локальное увеличение плотности состояний. 


\section{Список литературы}

[1] S. R. Elliot, M. Franz, "Colloquium: Majorana fermions in nuclear, particle, and solid-state physics", Rev. Modern Phys., 87:1 (2015), 137-163, arXiv: 1403.4976.

[2] M. Sato, S. Fujimoto, "Majorana fermions and topology in superconductors", J. Phys. Soc. Japan, 85:7 (2016), 072001, 32 pp., arXiv: 1601.02726.

[3] J. Alicea, "New directions in the pursuit of Majorana fermions in solid state systems", Rep. Progr. Phys., 75:7 (2012), 076501, 36 pp., arXiv: 1202.1293.

[4] S. Das Sarma, A. Nag, J.D. Sau, "How to infer non-Abelian statistics and topological visibility from tunneling conductance properties of realistic Majorana nanowires", Phys. Rev. B, 94:3 (2016), 035143, 17 pp.

[5] J.D. Sau, E. Demler, "Bound states at impurities as a probe of topological superconductivity in nanowires", Phys. Rev. B, 88:20 (2013), 205402, 6 pp.

[6] T. Karzig, G. Refael, F. von Oppen, "Boosting Majorana zero modes", Phys. Rev. X, 3:4 (2013), 041017, 16 pp.

[7] S. Das Sarma, J.D. Sau, T. D. Stanescu, "Splitting of the zero-bias conductance peak as smoking gun evidence for the existence of the Majorana mode in a superconductor-semiconductor nanowire", Phys. Rev. B, 86:22 (2012), 220506, 5 pp.

[8] C.-X. Liu, F. Setiawan, J.D. Sau, S. Das Sarma, "Phenomenology of soft gap, zero-bias peak, and zero-mode splitting in ideal Majorana nanowires", Phys. Rev. B, 96:5 (2017), 054520, 18 pp., arXiv: 1706.04573.

[9] C. Moore, T. D. Stanescu, S. Tewari, "Two-terminal charge tunneling: disentangling Majorana zero modes from partially separated Andreev bound states in semiconductor-superconductor heterostructures", Phys. Rev. B, 97:16 (2018), 165302, 14 pp., arXiv: 1611.07058.

[10] D. Chevallier, P. Simon, C. Bena, "From Andreev bound states to Majorana fermions in topological wires on superconducting substrates: a story of mutation", Phys. Rev. B, 88:16 (2013), 165401, 6 pp.

[11] Ю.Н. Демков, В.Н. Островский, Метод потенциалов нулевого радиуса в атомной физике, Изд-во Ленингр. гос. ун-та, Л., 1975.

[12] S. K. Adhikari, M. Casas, A. Puente, A. Rigo, M. Fortes, M. A. Solis, M. de Llano, A. A. Valladares, O. Rojo, "Linear to quadratic crossover of Cooper-pair dispersion relation", Physica C, 351:4 (2001), 341-348, arXiv: cond-mat/0008478.

[13] Y. Matsui, "Nucleon-trinucleon scattering with Yamaguchi potential", Progr. Theor. Phys., 104:5 (2000), 981-993.

[14] Yu. P. Chuburin, "Existence of Majorana bound states near impurities in the case of a small superconducting gap", Physica E, 89 (2017), 130-133.

[15] D. Sticlet, B. Nijholt, A. Akhmerov, "Robustness of Majorana bound states in the shortjunction limit", Phys. Rev. B, 95:11 (2017), 115421, 13 pp., arXiv: 1609.00637.

[16] P. San-Jose, J. Cayao, E. Prada, R. Aguado, "Majorana bound states from exceptional points in non-topological superconductors", Sci. Rep., 6 (2016), 21427, 13 pp.

Поступила в редакцию 4.09.2017, после доработки 28.02.2018 